\title{
Introduction
}

\section{Introduction to the Practice section}

Journal of Database Marketing \& Customer Strategy Management (2010) 17, 241. doi:10.1057/dbm.2010.22

Over the years, the Journal of Database Marketing has attempted to maintain a balance between papers that focus on specific issues of relevance to the Database Marketing and CRM practitioner community from an academic perspective and papers that highlight the practical experience of practitioners in this field, putting into practice the learnings either from academia or from their own experience.

This will continue.

With this issue, however, we are minded to make that division more overt, more formal. It is therefore our intention to make the difference between these two approaches even clearer by dividing the journal into Research and Practice sections. In all cases, we shall continue with our policy of rigorous peer review, and select only papers that we believe reflect current concerns in the field or advance thinking in specific areas.

However, we do recognise that the division between academic and practice reflects two very different knowledge bases: in the former, experimental and research; in the latter, experiential and results-focused. These approaches demand different ways of viewing the world, different standards of assessment and, to some degree, different levels of proof.

Welcome, therefore, to the Practice section - and please feed back any reaction you may have, positive or negative, to this new development.

Jane Fae Ozimek 